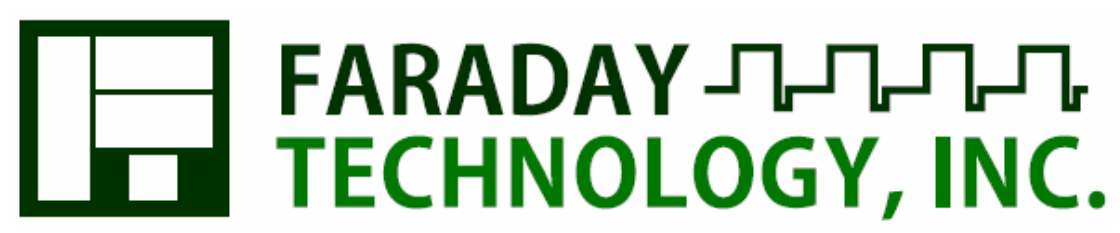

\title{
Multiphysics Modeling of the Tertiary Current Distribution in Pulse/Pulse-Reverse Electrochemical Processing of Microstructured Workpieces
}

\author{
Brian Skinn $^{\mathrm{a}}$, Alan C. West ${ }^{\mathrm{b}}$
}

a Faraday Technology, Inc., Englewood, OH, USA 45315

b School of Engineering and Applied Science, Columbia Univ., New York, NY, USA 10027

14 Oct 2019

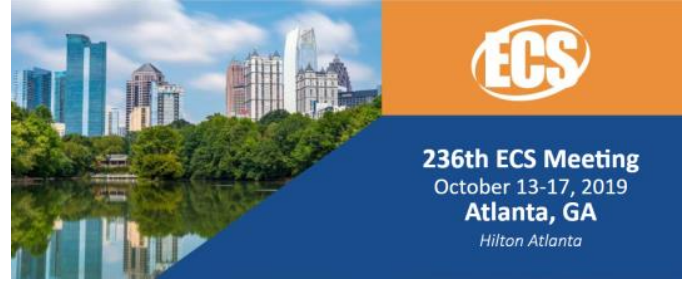




\section{Outline}

- Mass-Transfer Limitations in Electrochemical Surface Finishing

- Direct Current (Jacquet)

- Pulsed-Current

- $\mathrm{COMSOL}^{\circledR}$ Simulations

- 2-D System

- Empirical 'Surface-Occlusion' Model 


\section{Conventional Electrochemical Surface Finishing}

On some scale - All surfaces are rough:
1. $M^{0} \rightarrow M^{+}+e^{-}$
2. $x M+y H_{2} O \rightarrow M_{x} O_{y}+2 y H^{+}+2 y e^{-}$ $\rightarrow$ preferential removal of asperities $\leftarrow$

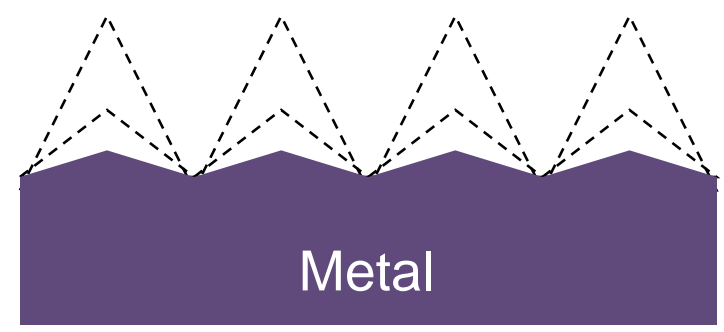

\section{Traditional ElectroFinishing:}

1. Smooth, bright surface finish achieved under diffusion limited conditions ${ }^{\dagger}$

$\rightarrow$ Viscous/concentrated acid electrolytes

2. For passive materials, electrolytes designed to mitigate oxide formation

$\rightarrow$ Oxide removal with fluoride or hydrofluoric acid (HF)

$\Rightarrow$ Non-aqueous to prevent oxide formation - not industrially practical

† P.A. Jacquet, Trans. Electrochem. Soc., 69629 (1936). 


\section{ElectroFinishing - Direct Current (DC)}

- Rectification:

- DC - Constant voltage

- Viscous salt film per Jacquet

- Passive (oxide) film formation

- Electrolyte:

- Concentrated/viscous acid

- Chilled to increase viscosity

- HF add to remove oxide $\dagger$ P.A. Jacquet, Trans. Electrochem. Soc., 69 629 (1936).
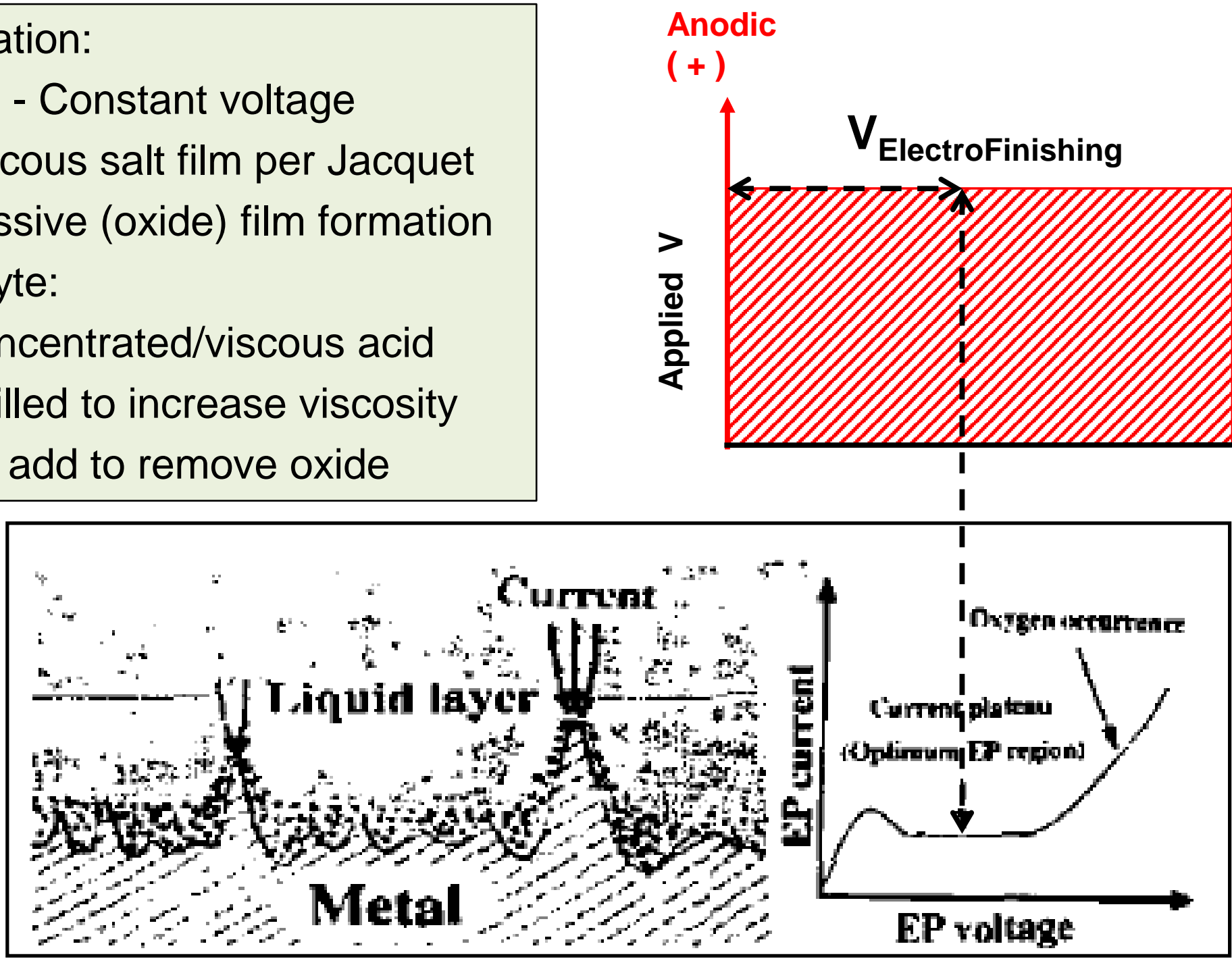

FARADAY ЛЛЛ TECHNOLOGY, INC. 


\section{ElectroFinishing - Pulsed Waveforms}

\section{TOOL}

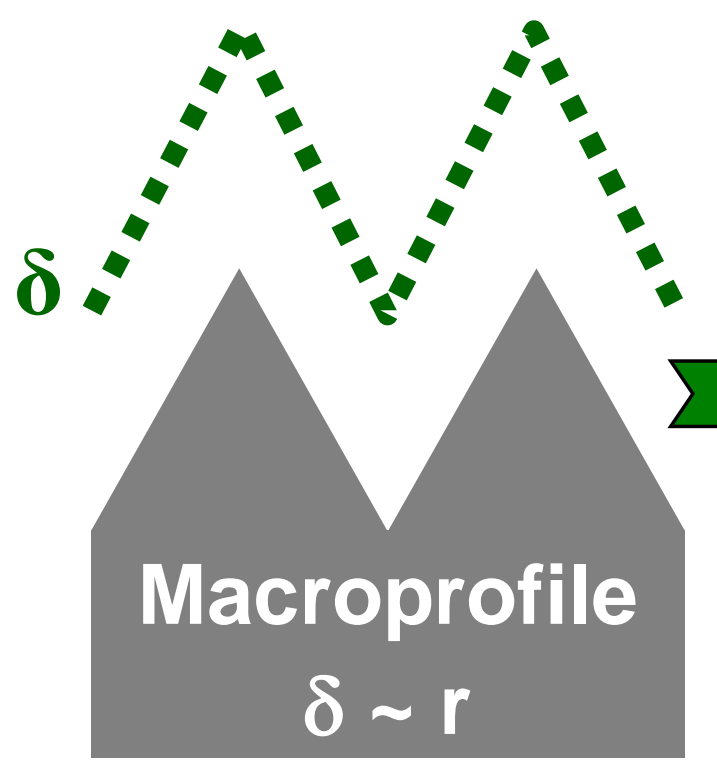

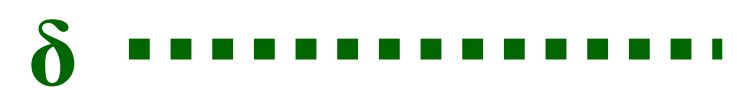

ठ

$(+)$
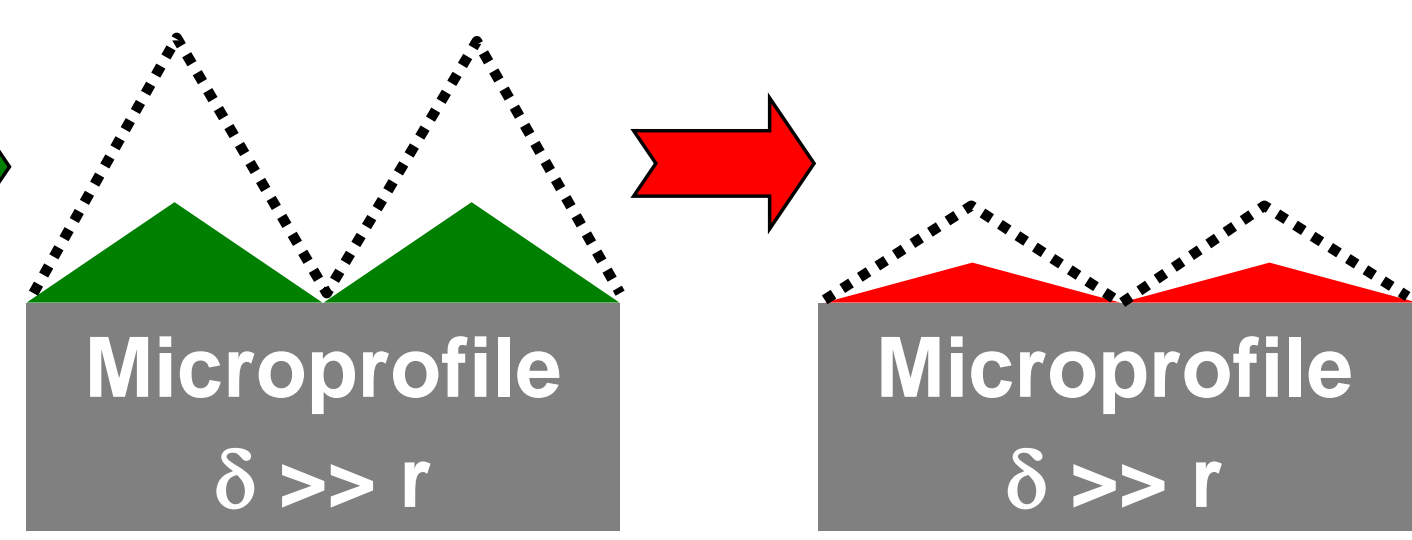

$\delta>r$
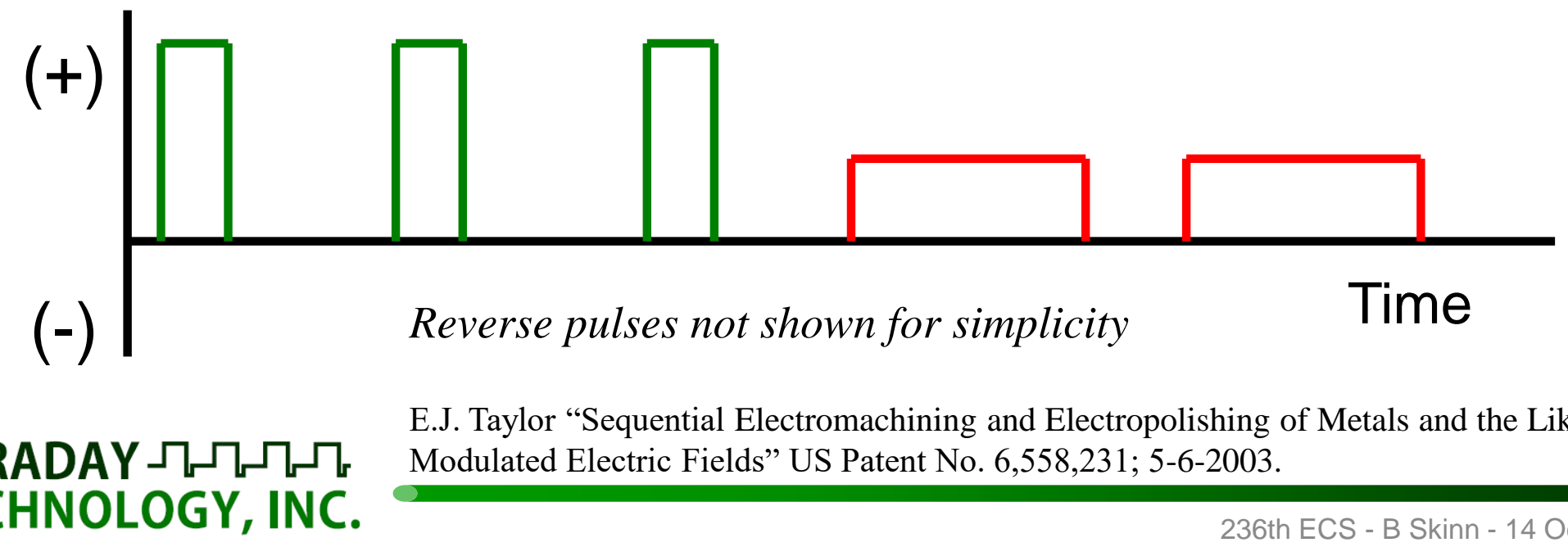

E.J. Taylor "Sequential Electromachining and Electropolishing of Metals and the Like Using Modulated Electric Fields" US Patent No. 6,558,231; 5-6-2003. 


\section{ElectroFinishing - Pulsed Waveforms}

\section{TOOL}

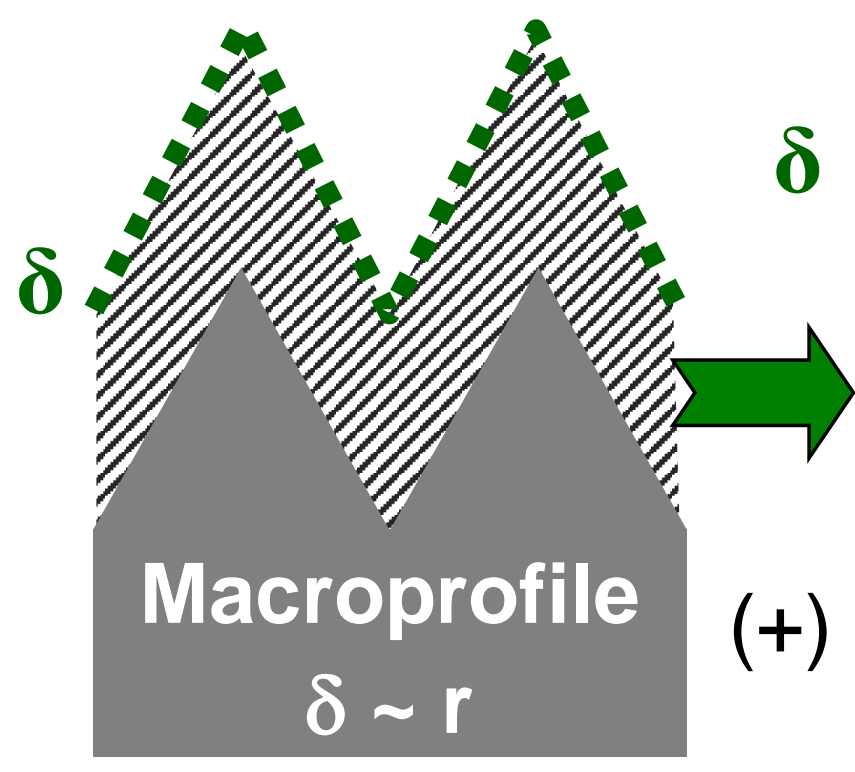

$(-)$

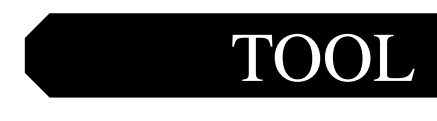

$(+)$

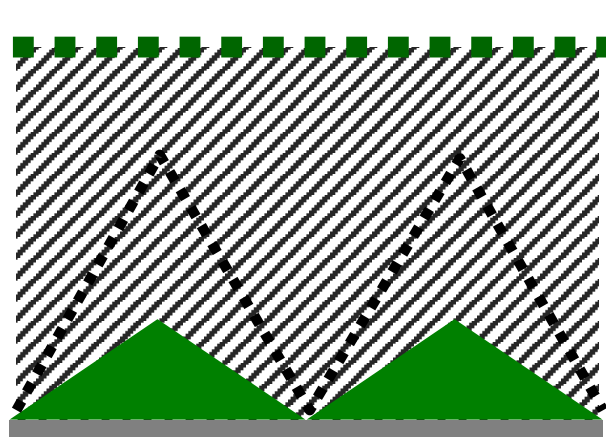

Microprofile

$$
\delta \gg r
$$

TOOL
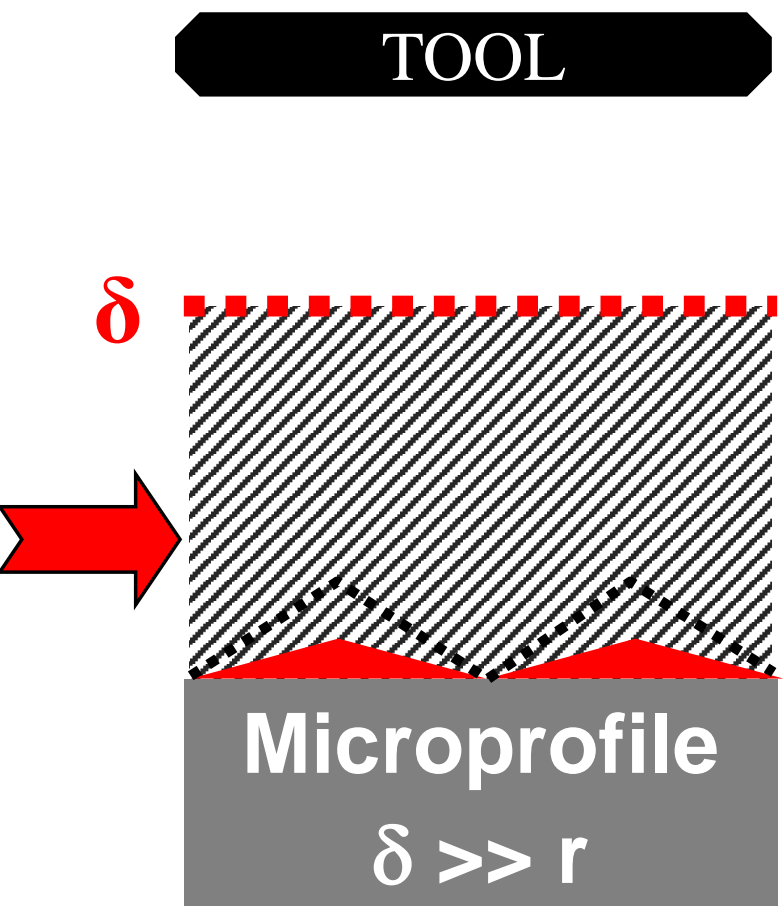

Microprofile

$$
\delta>r
$$
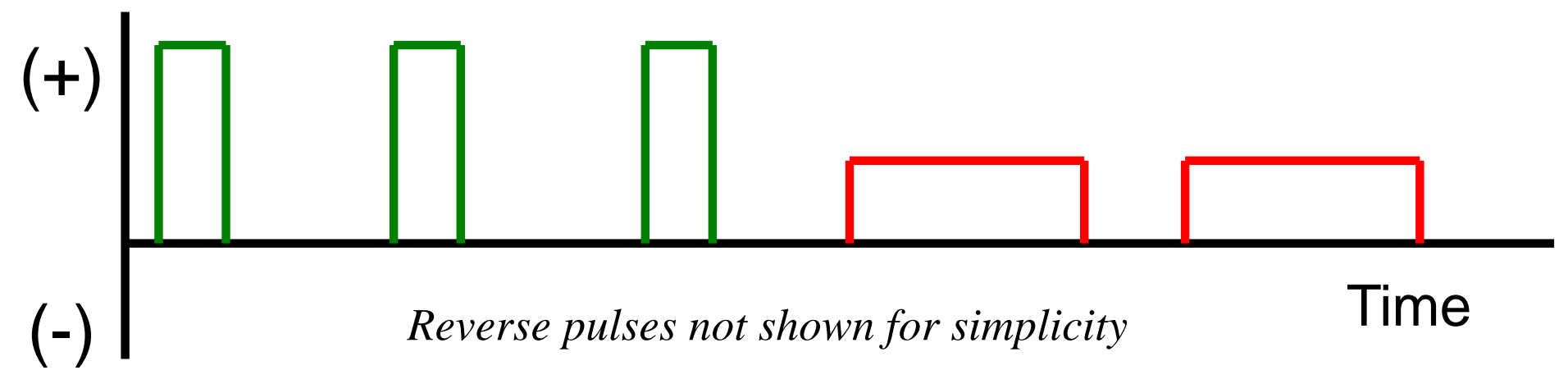

E.J. Taylor "Sequential Electromachining and Electropolishing of Metals and the Like Using Modulated Electric Fields" US Patent No. 6,558,231; 5-6-2003.

FARADAY תЛЛת TECHNOLOGY, INC. 


\section{P/PR Waveform Sequencing - SS316 Tubes}

$>$ Pulse/Pulse Reverse

o Initial $R_{a} \sim 1 \mu \mathrm{m}$

o Waveform \#1

$\rightarrow$ Short forward pulses

$\rightarrow$ Reverse (cathodic) pulses

$\circ$ After $30 \mathrm{~s}: \mathrm{R}_{\mathrm{a}} \sim 0.2 \mu \mathrm{m}$

o Waveform \#2

$\rightarrow$ Long forward pulses

$\rightarrow$ Reverse (cathodic) pulses

o After additional $15 \mathrm{~s}: \mathrm{R}_{\mathrm{a}} \sim 0.05 \mu \mathrm{m}$
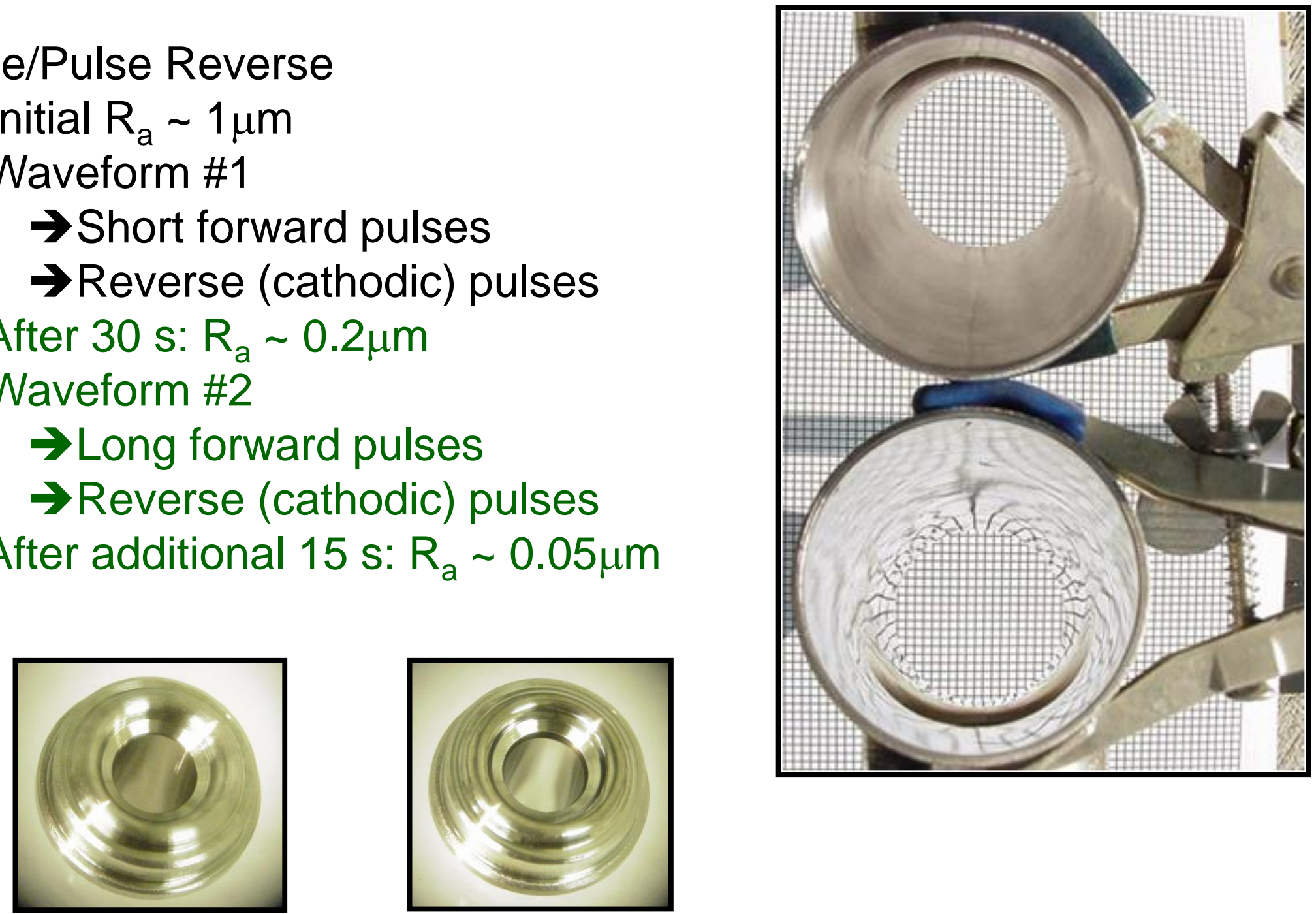

FARADAY ЛЛЛ TECHNOLOGY, INC. 


\section{P/PR Waveform Sequencing - AM Ti Alloy}

Initial Trial

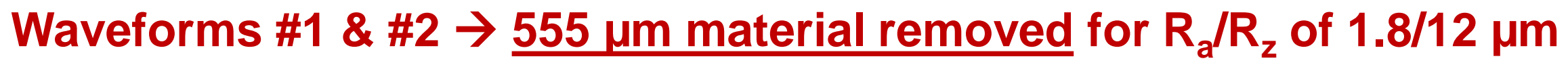

Improved Waveform Sequence
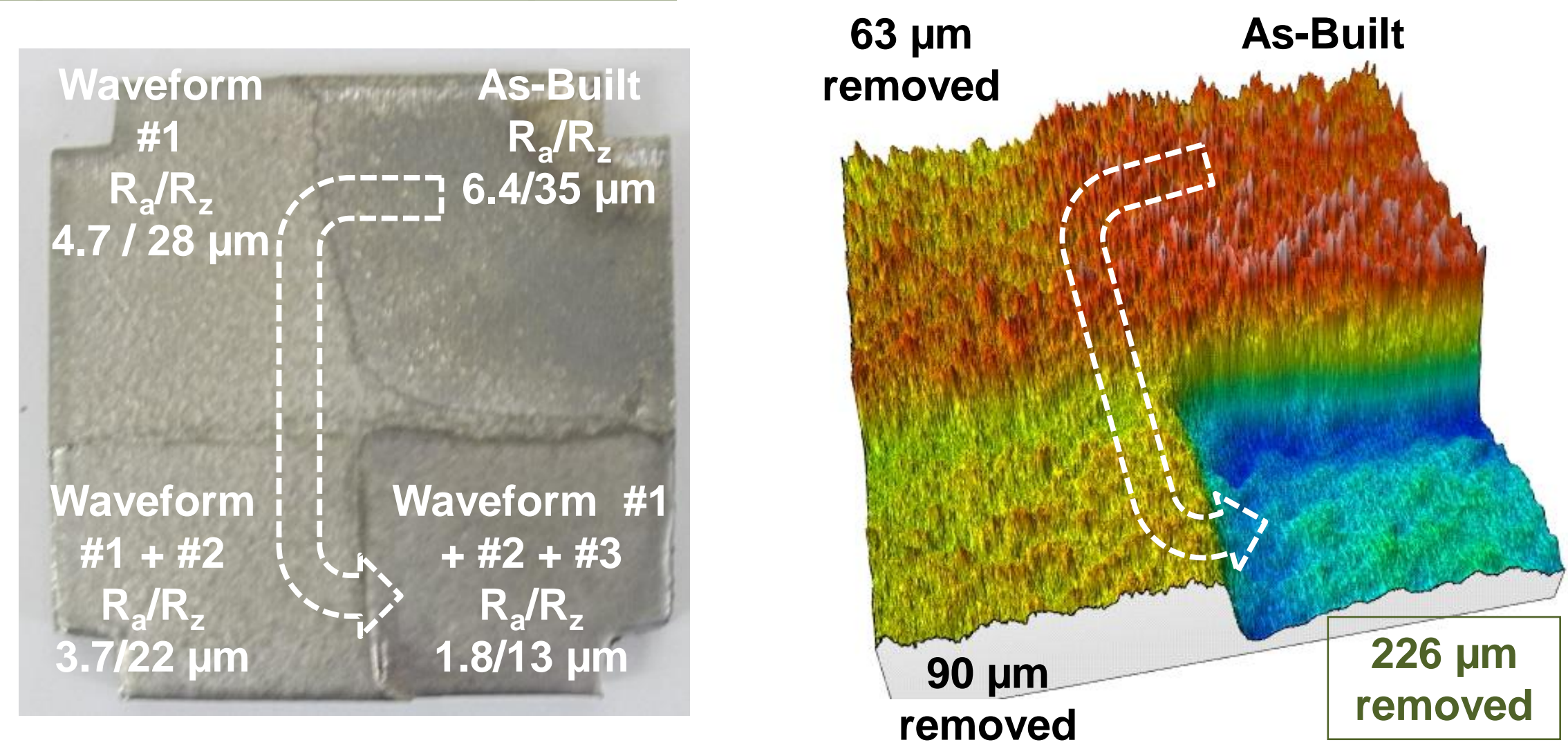

FARADAY ЛПЛ TECHNOLOGY, INC. 


\section{P/PR Waveform Sequencing - AM Hastelloy-X}

$>$ CHALLENGE: improve $R_{\mathrm{a}}$ to $2 \mu \mathrm{m}$ while minimizing material removal

- Waveform 1:

- Initial rapid $\Delta \mathrm{R}_{\mathrm{a}} / \Delta$ material removed until $\sim 6 \mu \mathrm{m} \mathrm{R}_{\mathrm{a}}$ reached

- Decrease in $\Delta \mathrm{R}_{\mathrm{a}} / \Delta$ material removed after $\sim 6 \mu \mathrm{m} \mathrm{R}_{\mathrm{a}}$ reached

- Waveform 2:

- Initially slow $\Delta \mathrm{R}_{\mathrm{a}} / \Delta$ material removed until $\sim 6 \mu \mathrm{m} \mathrm{R}_{\mathrm{a}}$ reached

- Increase in $\Delta R_{a} / \Delta$ material removed after $\mathrm{R}_{\mathrm{a}}<6 \mu \mathrm{m}$

$>$ SOLUTION: Initiate process with Waveform 1 (4 min) until $R_{a} \sim 6 \mu \mathrm{m}$, then switch to Waveform 2 (1 min) until next critical $R_{a}$ $\sim 1.8$ with $80 \mu \mathrm{m}$ removed.

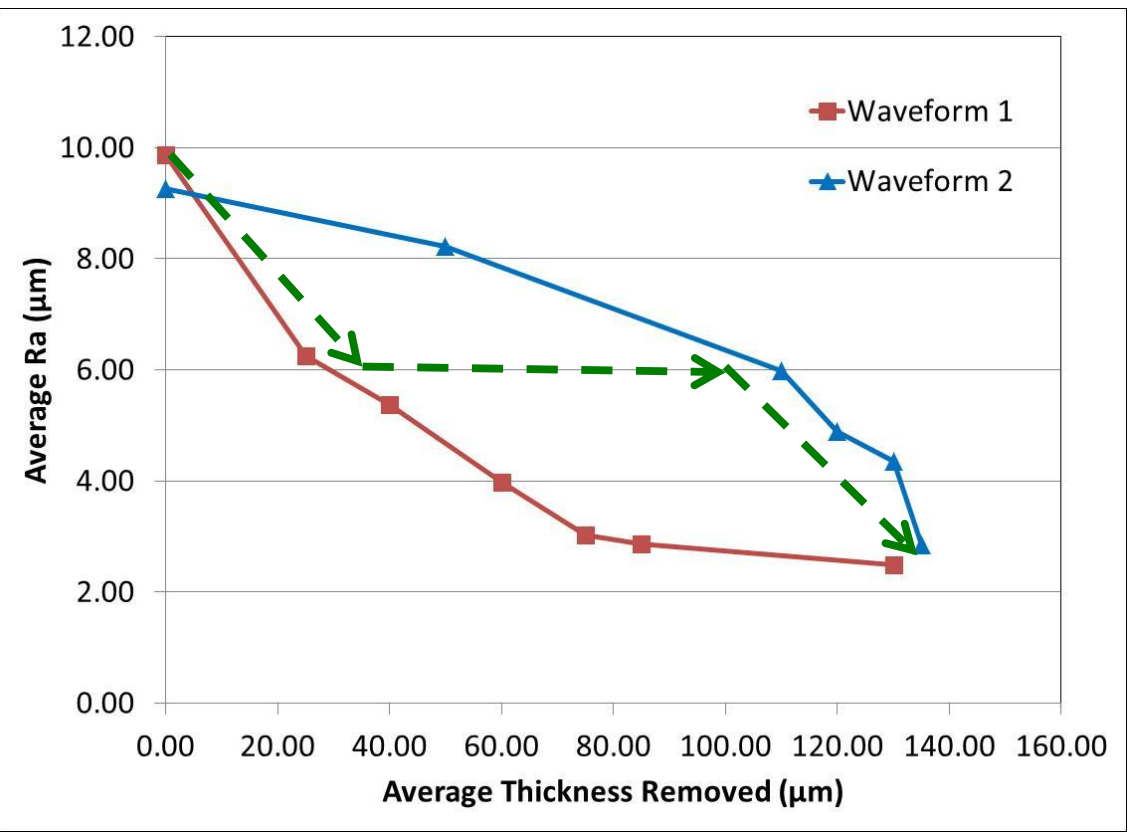

$\rightarrow$ Tune process by waveform sequencing in order to achieve desired surface finish while minimizing material removal $\leftarrow$ 


\section{FARADAY תЛתЛ
TECHNOLOGY, INC.}

\section{COMSOL ${ }^{\circledR}$ Simulations}

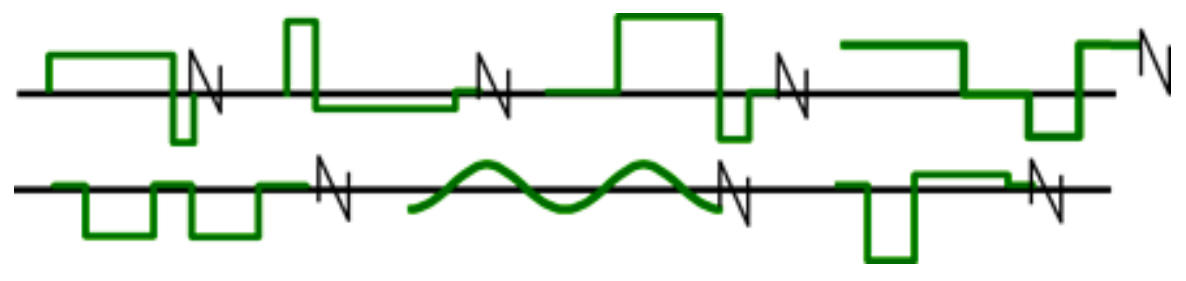




\section{Physical Phenomena in Electrochemical Processes}

- Primary Current Distribution

- Laplace equation (Ohmic/resistive potentials)

- Equilibrium reaction potentials

- Secondary Current Distribution

- Kinetic polarization (Butler-Volmer/Tafel)

- Capacitive polarization (Helmholtz/Gouy-Chapman/Stern/...)

- Tertiary Current Distribution

- Homogeneous

- Convective \& diffusive currents

- Ion migration

- Concentration dependency of diffusivity, etc.

- Heterogeneous

- Concentration overpotential

- Jacquet effects - 'surface-occlusion saturation model'

- Other

- Carbon deposits/residues

- Passive oxide formation

FARADAY ת ת TECHNOLOGY, INC. 


\section{COMSOL ${ }^{\circledR}$ - Model Geometry \& Parameters}

\section{Geometric Parameters}

$$
\begin{array}{cl}
h_{\text {peak }} & =5 \mu \mathrm{m} \\
w_{\text {peak }} & =6 \mu \mathrm{m} \\
w_{\text {spacing }} & =30 \mu \mathrm{m} \\
\delta_{H} & =30 \mu \mathrm{m}
\end{array}
$$

\section{Physicochemical Parameters}

$$
\begin{array}{ccc}
D & = & 10^{-10} \mathrm{~m}^{2} / \mathrm{s} \\
C_{\text {sat }} & = & 0.4 \mathrm{M} \\
\varphi & =100 \%
\end{array}
$$

\section{Electrochemical Parameters}

$\begin{array}{ccc}z & = & 2 \\ \alpha_{M, a} & = & 1.5 \\ \alpha_{M, c} & = & 0.5 \\ j_{o, M} & = & 2 \mathrm{~mA} / \mathrm{cm}^{2}\end{array}$

Edge of

Hydrodynamic

Boundary Layer

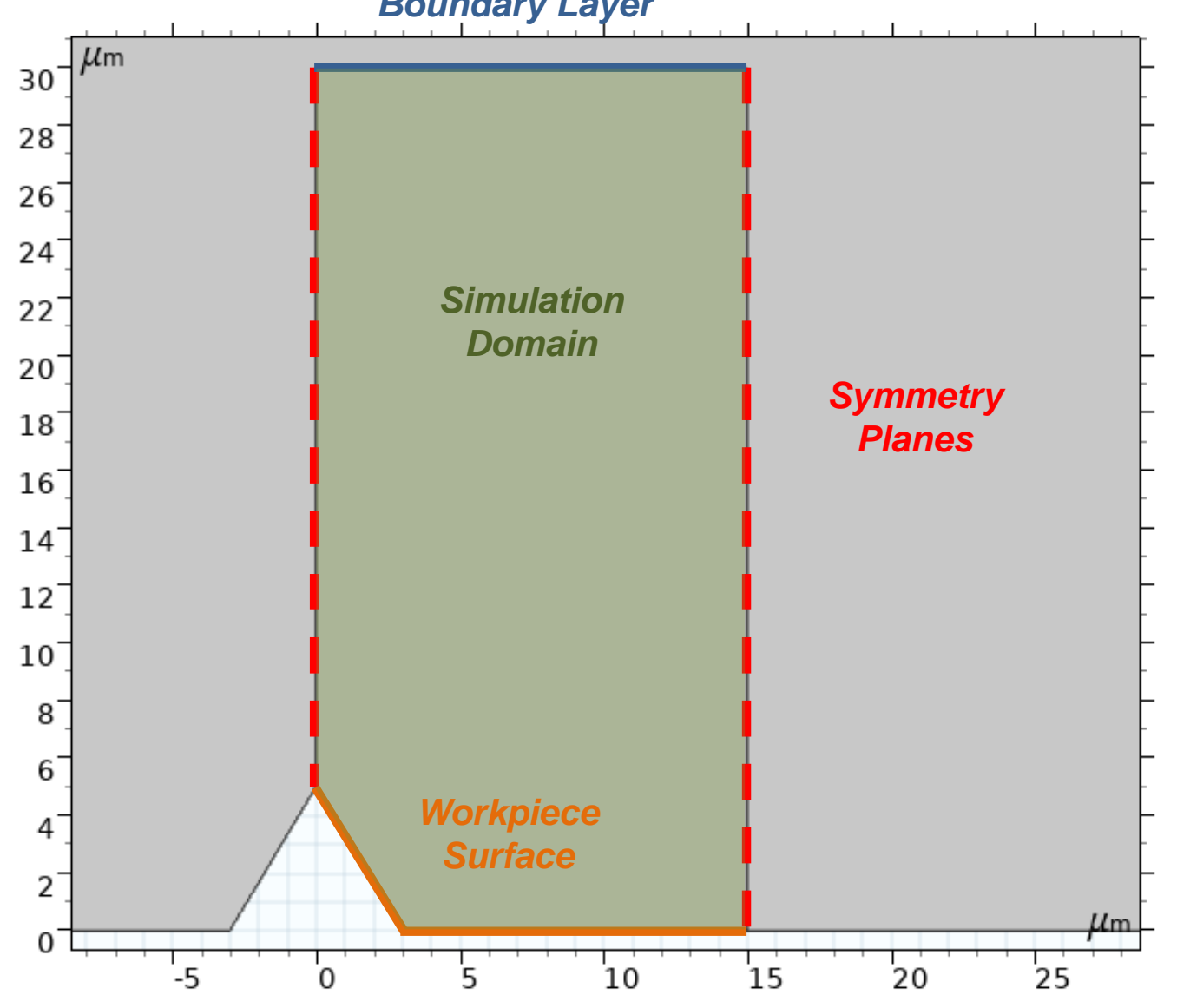




\section{COMSOL $^{\circledR}$ - Pulsed Waveforms}

- $\operatorname{Period}(T)=$ On-time $\left(t_{\text {on }}\right)+$ Off-time $\left(t_{\text {off }}\right)$

- Frequency $(v)=1 / T$

- Duty Cycle $(\lambda)=t_{\mathrm{on}} / T$

- Peak Current Density $\left(j_{p}\right)$

- Average Current Density $\left(j_{a}=\lambda j_{p}\right)$

\section{Workpiece Boundary Condition}

$$
\left.\frac{\partial C}{\partial y}\right|_{y=0}=\left\{\begin{array}{cl}
-\frac{j_{p}}{n F D} & \text { during } t_{o n} \\
0 & \text { during } t_{o f f}
\end{array}\right.
$$

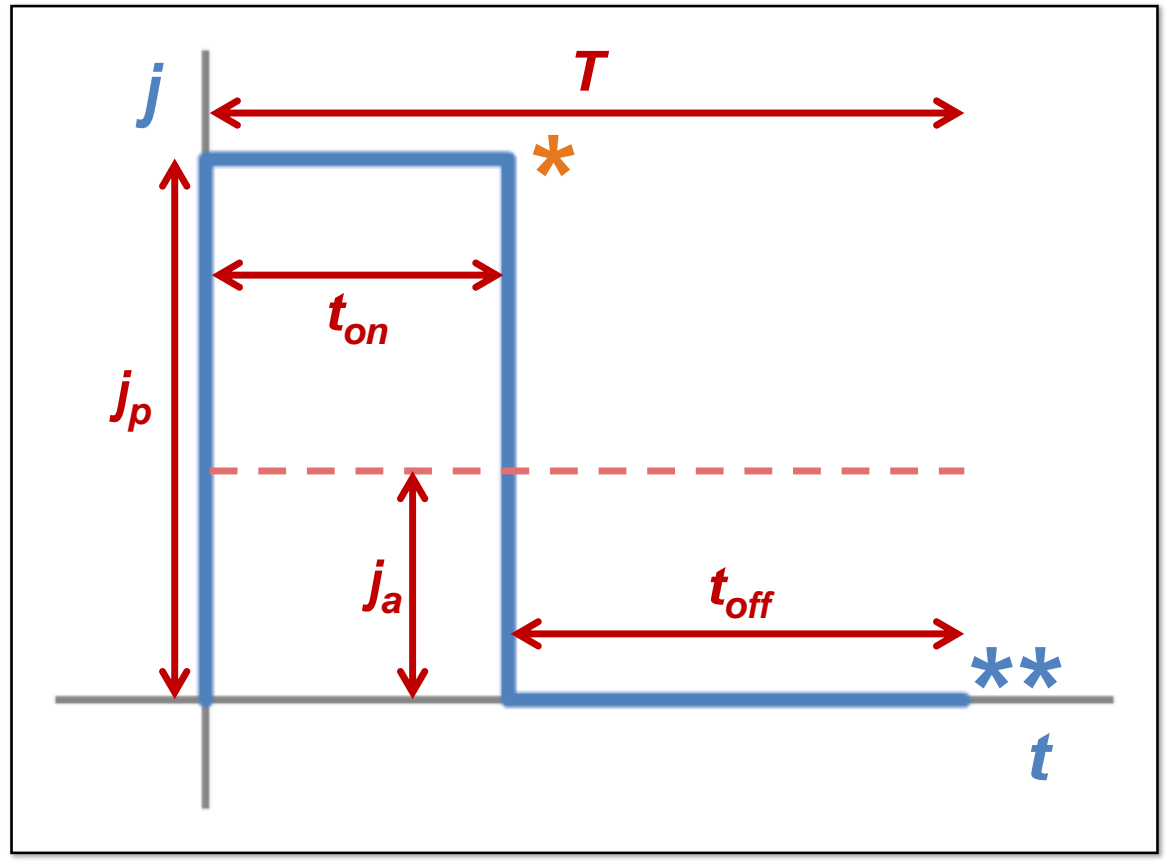

\section{Concentration Profiles - Color Legend}

* Profile at end of on-time $\quad * *$ Profile at end of off-time

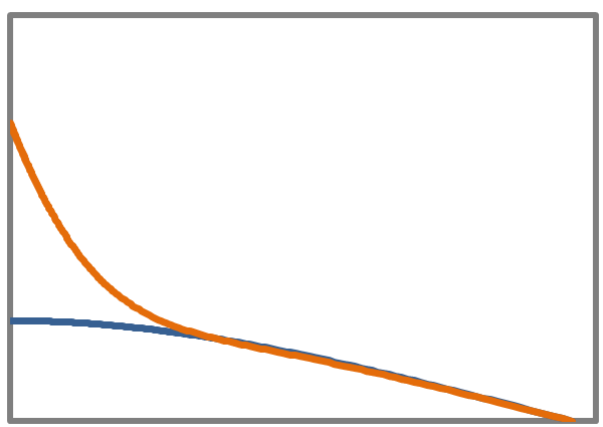




\section{COMSOL $^{\circledR}$ - Empirical Surface Saturation Model}
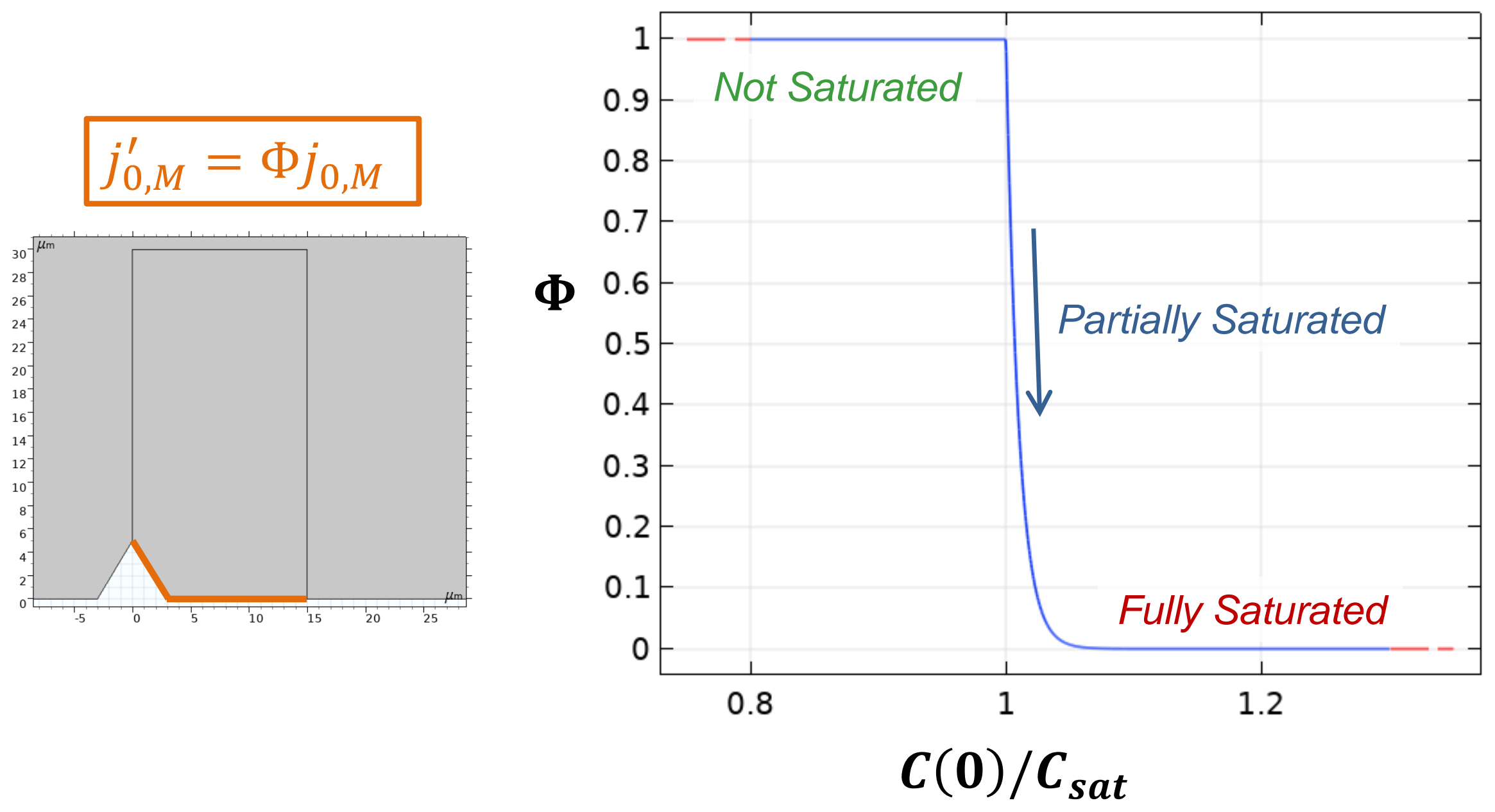


\section{Results - Effect of Tertiary Current Distribution}

Tertiary Current Distribution ENABLED

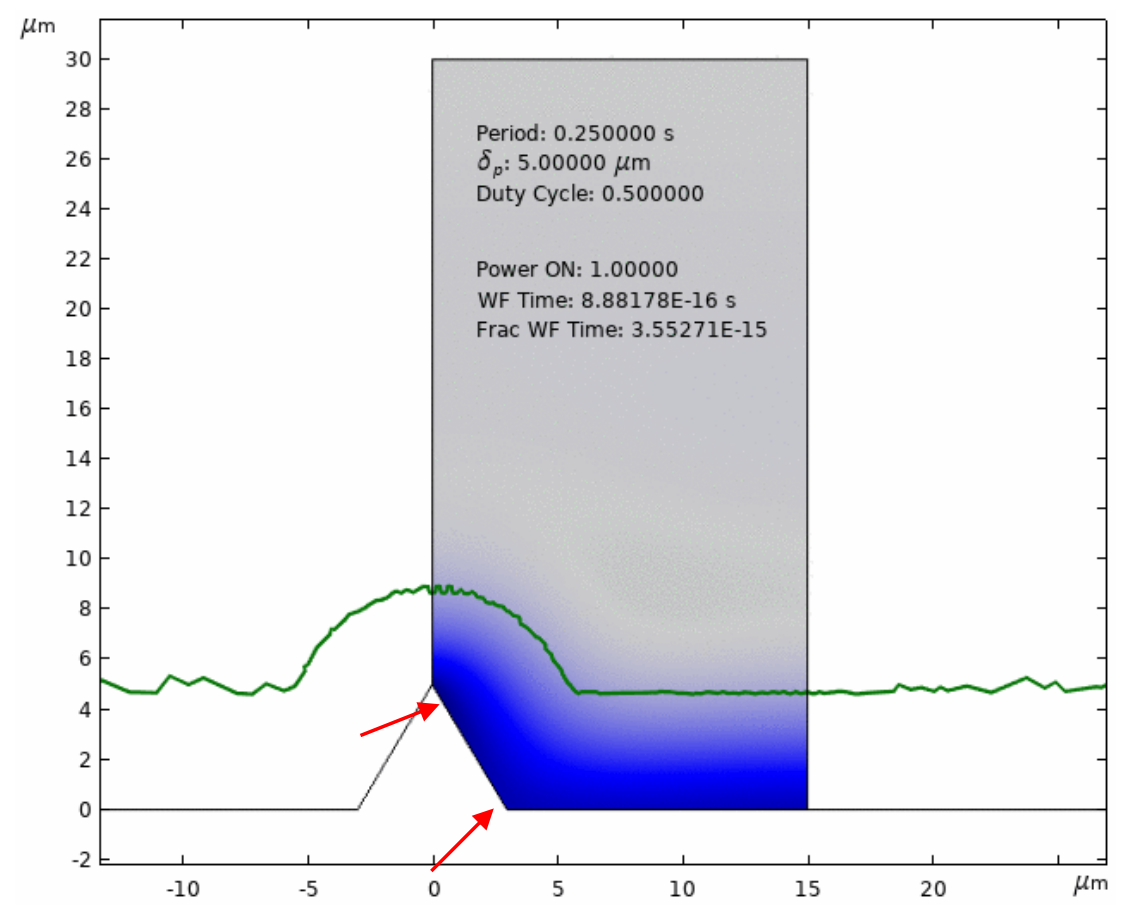

Tertiary Current Distribution DISABLED

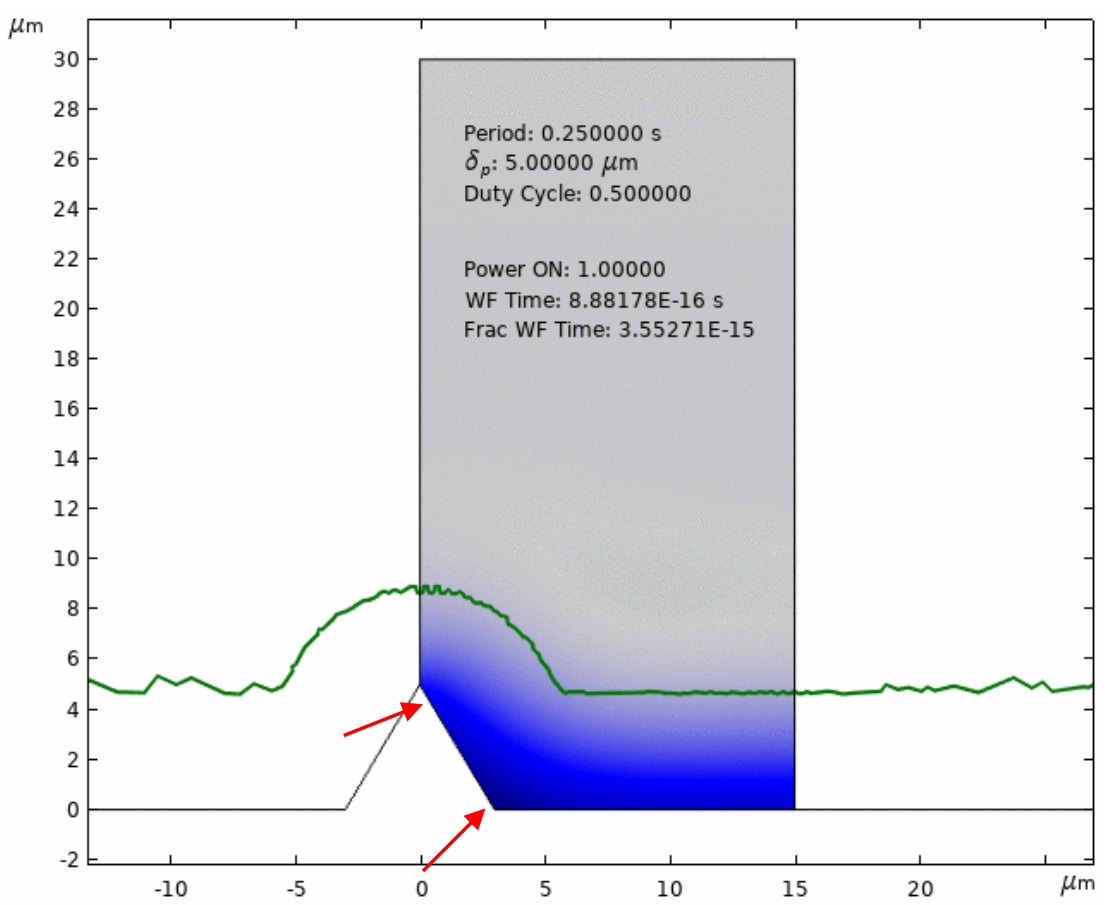




\section{Results - Effect of Tertiary Current Distribution}

Tertiary Current Distribution ENABLED

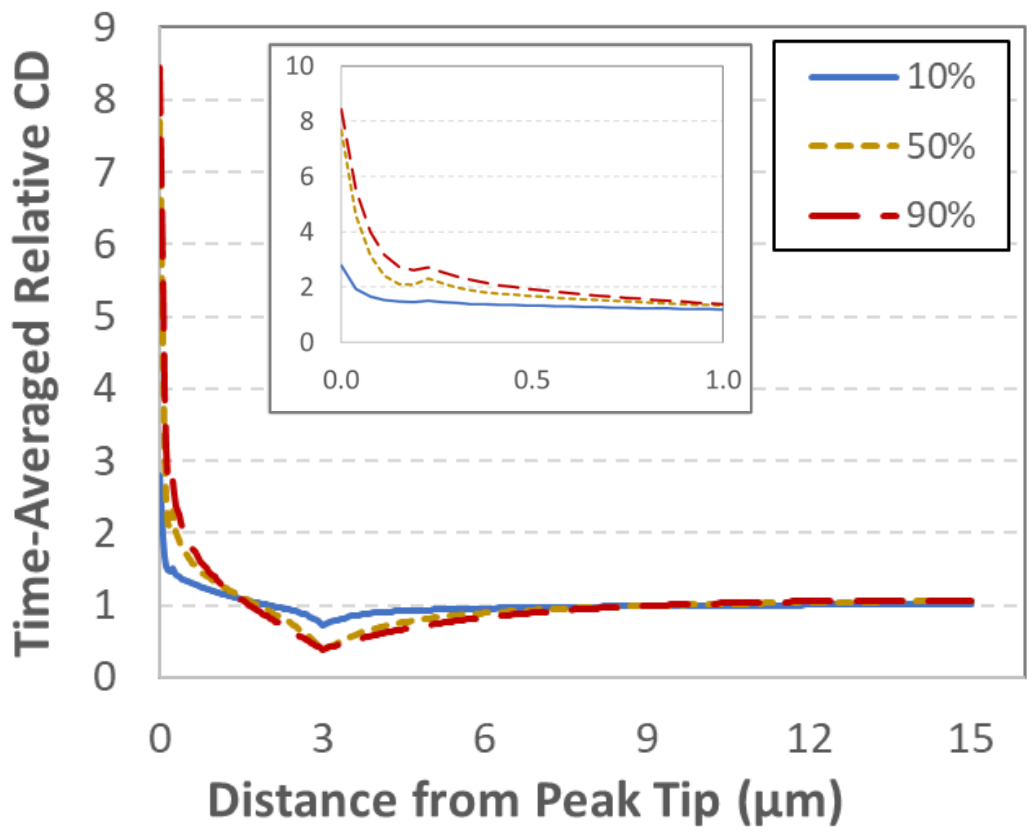

Tertiary Current Distribution DISABLED

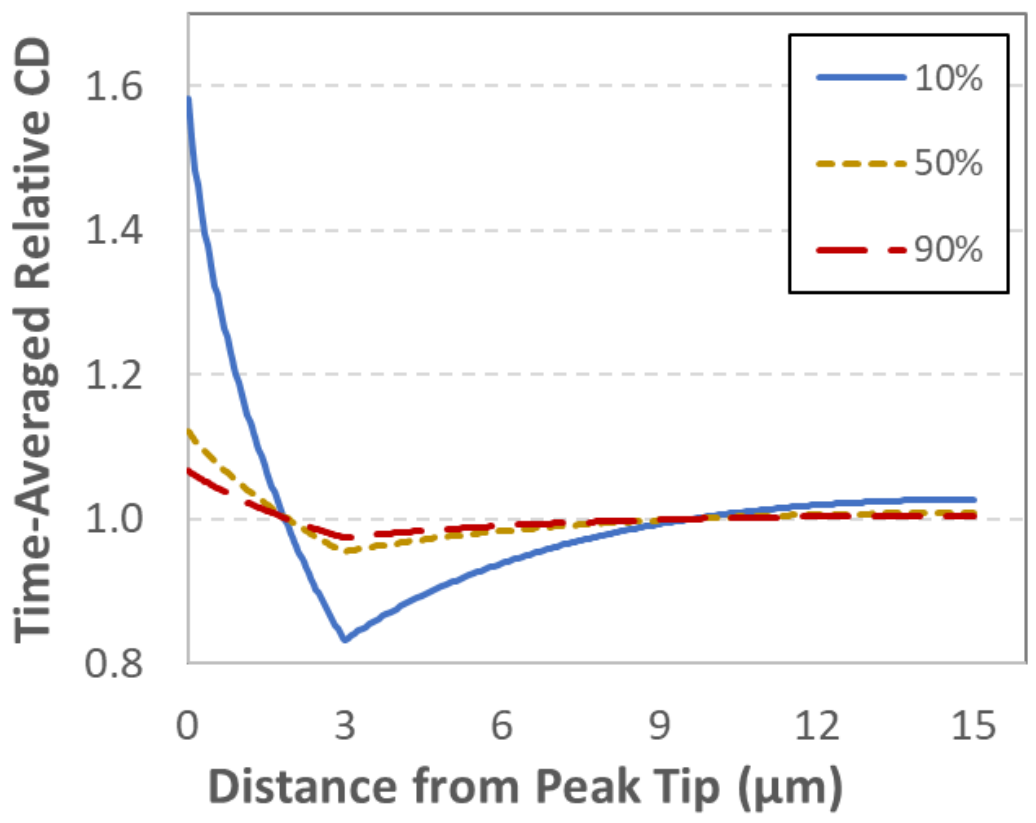




\section{Results - Function of Feature Size}
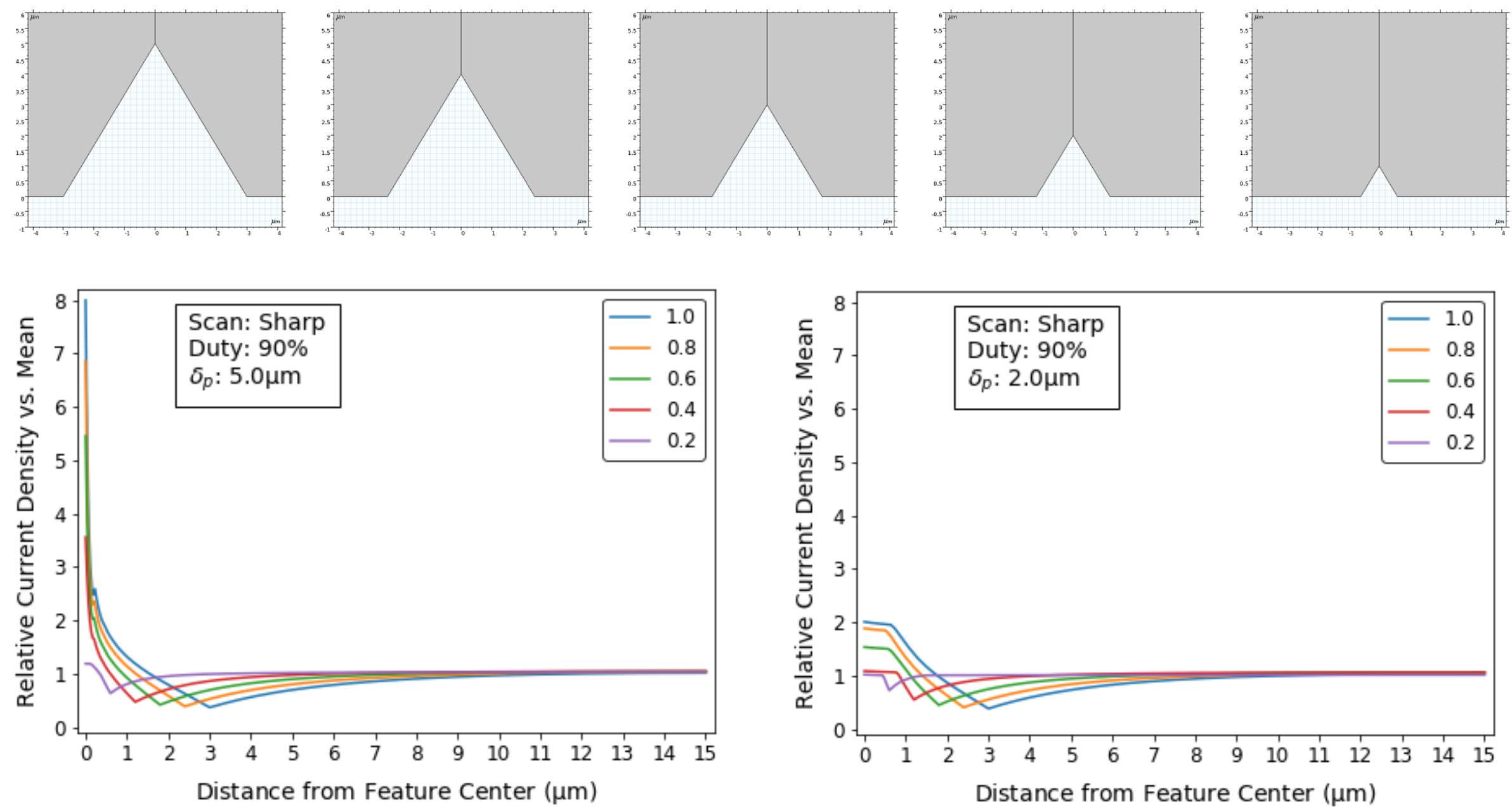

FARADAY תת ת TECHNOLOGY, INC. 


\section{Summary}

- Empirical treatment of mass-transfer limitations with a surface-occlusion model successfully represents qualitative features of experimental observations

- Multiphysics modeling has the potential to provide guidance for pulsed-waveform selection

- Optimal pulse waveforms arise from a complex interaction of geometrical, physicochemical and electrochemical
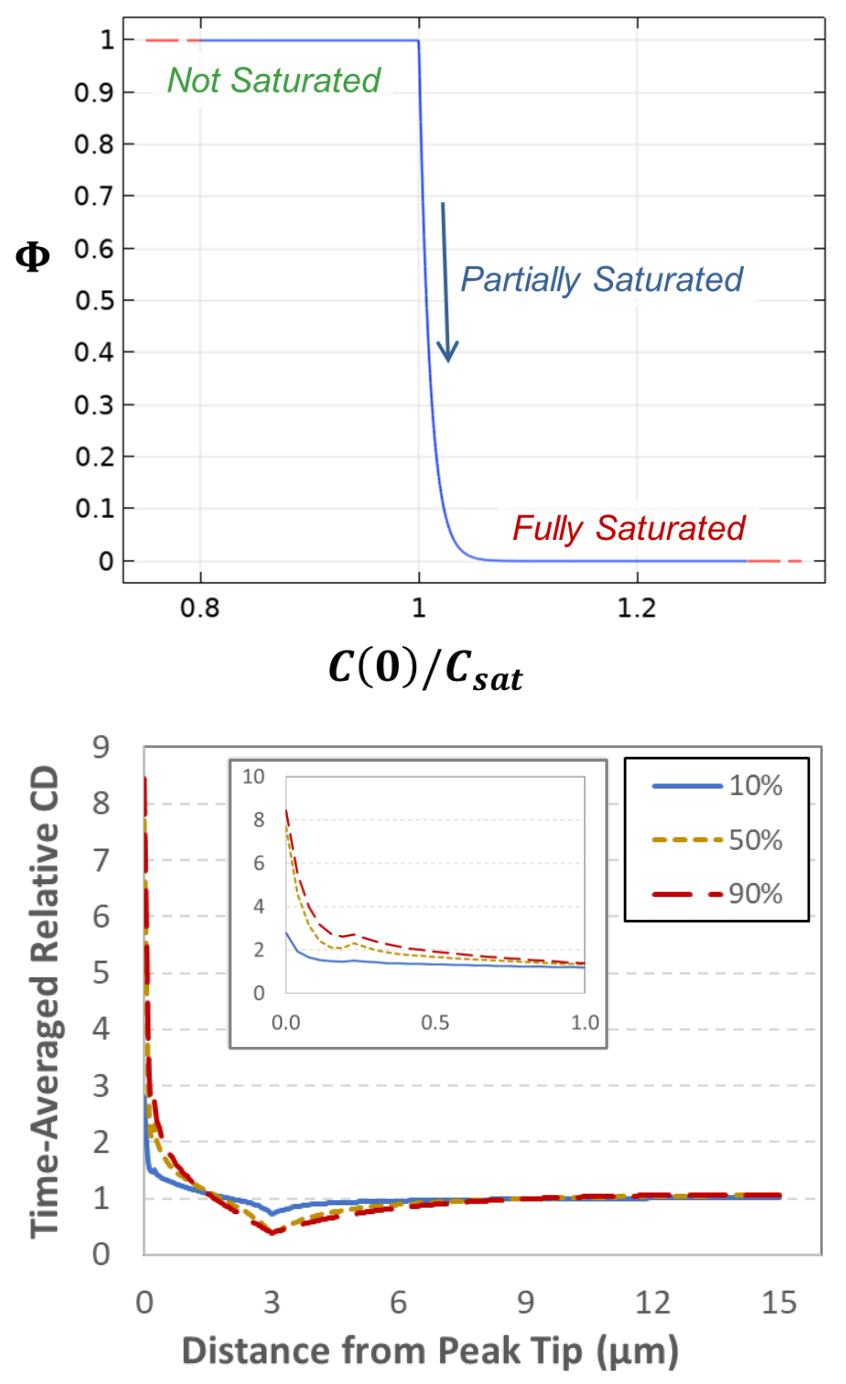


\section{Acknowledgment}

- This material is based upon work/resources supported by:

- US Army SBIR Grant \#W15QKN-18-C-0010

- Commercial Partners

- Faraday Corporate

- Contact Information:

Brian T. Skinn

Ph: +1937 8367749

Email: BrianSkinn@FaradayTechnology.com

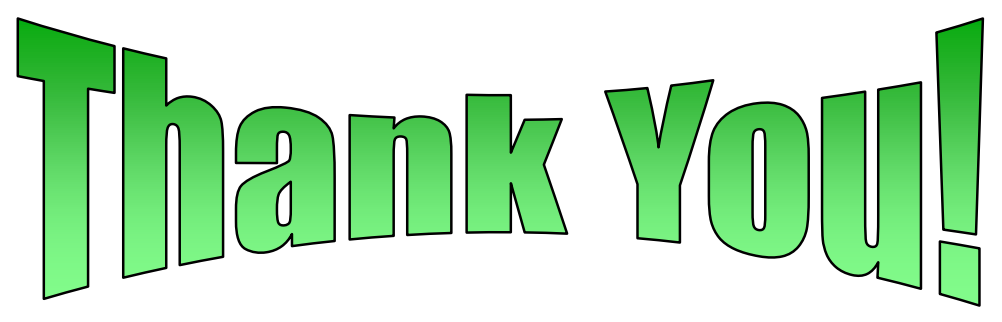

